\title{
COMMENTARY
}

\section{Improving ascertainment and communication of prognosis in intracerebral hemorrhage}

\author{
Emily J Gilmore and Kevin N Sheth
}

See related research by Ji et al., http://ccforum.com/content/17/6/R275

\begin{abstract}
Prognostication after intracerebral hemorrhage remains a significant challenge for the field of neurocritical care. Despite several available tools that can predict mortality and, to some degree, functional outcomes, deciding which prediction score to use and how best to translate the resultant population-based value to the individual level is not always clear. As more and more scores are published, we need to give due attention to the qualitative aspect of prognostication and explore how best to move this critical aspect of our field forward.
\end{abstract}

For more than a decade, researchers in neurocritical care and stroke have attempted to develop prediction models for patients with intracerebral hemorrhage (ICH) that can be used to accurately prognosticate across varied patient populations. In this issue of Critical Care, Ji and colleagues [1] join the ranks with their report on a refined prediction model using a cohort from the China National Stroke Registry (CNSR).

Central to the field of critical care neurology is the extremely difficult challenge for practitioners not only to prognosticate but to prognosticate well. Communicating the dynamic, often intangible, concept that is prognosis is paramount, as is doing so in a way that is patientcentered and specific. Often, it is not the far ends of the spectrum that are tough to foretell, but rather the vast space that lies between.

Prior studies in acute severe brain disease have shown that neurointensivists are better at predicting poor outcome than good outcome [2], but tend to overestimate poor outcome and underestimate good outcome [3]. In addition, a recent study in ischemic stroke suggests that

\footnotetext{
* Correspondence: emily.gilmore@yale.edu

Division of Neurocritical Care and Emergency Neurology, Department of Neurology, Yale School of Medicine, Yale-New Haven Hospital, PO Box 208018, New Haven, CT 06520-3221, USA
}

scoring systems may be more accurate than the prognostic estimation of practicing clinicians [4]. This work gives rise to the hypothesis that formal, rigorously tested, and validated scores may be superior to our judgment and may facilitate the development of a quantitative framework for prognostication.

Ji and colleagues' proposed score, the ICH-functional outcome score (ICH-FOS), is a 16-point scale that consists of easily obtained admission variables and variables that have been shown to be predictive of poor outcome in existing scores. The ICH-FOS, which includes age, admission National Institute of Health Stroke Scale (NIHSS) and Glasgow Coma Scale (GCS) scores, admission glucose, ICH location, hematoma volume, and intraventricular extension, sheds some light on the 'crystal ball' question for patients with ICH. In head-to-head comparisons with eight other prediction scores, the ICH-FOS conveyed similar or better accuracy in predicting shorter-term outcomes and was superior in predicting longer-term outcomes across different modified Rankin Scale scores in the CNSR cohort.

There is no paucity of literature conveying that donot-resuscitate orders limit care and contribute to worse outcomes in patients with ICH [5-8]. Unfortunately, all available scores are hindered by this self-fulfilling prophecy and would certainly be more optimistic if they were adjusted for earlier deaths due to withdrawal or lessintensive forms of treatment. The only way to precisely define prognosis in $\mathrm{ICH}$ would be to assess it in a cohort in which all patients received full support, irrespective of the perceived probable outcome. The CNSR cohort used in this study had an overall low rate of withdrawal of medical care $(12.4 \%)$, perhaps allowing us to get closer to overcoming the self-fulfilling prophecy prejudice and take a look at the natural recovery of this disease process.

In light of the improved predication accuracy, the CNSR cohort is a relatively 'healthy' group of ICH patients when compared with other cohorts used to derive 
and validate previous prognostic models. The majority of patients had admission GCS scores of at least to 9 as well as NIHSS scores of not more than 17 . Only $1 \%$ of patients were on anticoagulation at the time of admission, and the majority of patients had bleeds of not more than $28 \mathrm{~cm}^{3}$, and only about $30 \%$ had intraventricular extension, the volume of which was not quantified. Conceivably the authors found their prediction tool to be more accurate than the other eight prediction tools because their cohort differed significantly from the derivation cohorts of these other prediction tools. Thus, it is prudent that ICH-FOS be corroborated in Western populations before it is ready for prime time.

Finally, it is not just about the accuracy of numbers and percentages, but how we translate them into the everyday conversations we have with patients and families. Now more than ever, we are in an era of prognostic uncertainty for acute brain disease. There are no specific guidelines in neurology or neurocritical care for how to extrapolate scores to an individual level. For many, that is the art of medicine. However, given the conflicting data on how good we are at painting a balanced picture, we need to embrace the uncertainty and strive to do better. In a recent publication, Holloway and colleagues [9] challenge us as a field to be not only more humble but more scientific and methodical in our prognostication and communication approach. Raising the research bar on both a quantitative and qualitative level will improve our ability not only to prognosticate but hopefully to have more data-driven discussions with patients and families about mortality and, more importantly, functional and cognitive outcomes. Ji and colleagues add meaningfully to our prediction armamentarium with the ICH-FOS, but there is still much work to be done. It is unlikely that scores will ever tell the whole story. Perhaps research efforts understanding and comparing the prognostic assessments of various team members (that is, physicians and nurses) will complement our quantitative approaches and improve our clinical acumen in this vital facet of our field.

With several clinical studies under way in acute ICH management - Minimally Invasive Surgery plus rt-PA for ICH Evacuation, phase III (MISTIE-III), Clot Lysis Evaluating Accelerated Resolution of Intraventricular Hemorrhage, phase III (CLEAR-III), Antihypertensive Treatment of Acute Cerebral Hemorrhage (ATACH-II), and High-Dose Deferoxamine in Intracerebral Hemorrhage (HiDef) - as well as continued progress in the fields of rehabilitation and restorative medicine, the art of outcome prediction will remain a moving target. Although aggressiveness of treatment may be controversial, and intractable conditions can be challenging in the face of limited evidence-based guidance, treatment should not be relegated to the nihilistic fate of the self-fulfilling prophecy.

\section{Abbreviations}

CNSR: China National Stroke Registry; GCS: Glasgow Coma Scale;

ICH: Intracerebral hemorrhage; ICH-FOS: Intracerebral hemorrhage-functional outcome score; NIHSS: National Institute of Health Stroke Scale.

\section{Competing interests}

The authors declare that they have no competing interests.

\section{Authors' contributions}

EJG drafted the article and KNS revised it for critical content. Both authors read and approved the final manuscript.

\section{Published: 09 Dec 2013}

\section{References}

1. Ji R, Shen H, Pan Y, Wang P, Liu G, Wang Y, Li H, Zhao X, Wang Y: A novel risk score to predict 1-year functional outcome after intracerebral hemorrhage and comparison with existing scores. Crit Care 2013, 17:R275.

2. Finley Caulfield A, Gabler L, Lansberg MG, Eyngorn I, Mlynash M, Buckwalter MS, Venkatasubramanian C, Wijman CA: Outcome prediction in mechanically ventilated neurologic patients by junior neurointensivists. Neurology 2010, 74:1096-1101.

3. Kaufmann MA, Buchmann B, Scheidegger D, Gratzl O, Radu EW: Severe head injury: should expected outcome influence resuscitation and first-day decisions? Resuscitation 1992, 23:199-206.

4. Saposnik G, Cote R, Mamdani M, Raptis S, Thorpe KE, Fang J, Redelmeier DA, Goldstein LB: JURaSSiC: accuracy of clinician vs risk score prediction of ischemic stroke outcomes. Neurology 2013, 81:448-455.

5. Hemphill JC 3rd, Newman J, Zhao S, Johnston SC: Hospital usage of early do-not-resuscitate orders and outcome after intracerebral hemorrhage. Stroke 2004, 35:1130-1134.

6. Creutzfeldt CJ, Becker KJ, Weinstein JR, Khot SP, McPharlin TO, Ton TG, Longstreth WT Jr, Tirschwell DL: Do-not-attempt-resuscitation orders and prognostic models for intraparenchymal hemorrhage. Crit Care Med 2011, 39:158-162

7. Zahuranec DB, Morgenstern LB, Sanchez BN, Resnicow K, White DB, Hemphill JC 3rd: Do-not-resuscitate orders and predictive models after intracerebral hemorrhage. Neurology 2010, 75:626-633.

8. Becker KJ, Baxter AB, Cohen WA, Bybee HM, Tirschwell DL, Newell DW, Winn $H R$, Longstreth WT Jr: Withdrawal of support in intracerebral hemorrhage may lead to self-fulfilling prophecies. Neurology 2001, 56:766-772.

9. Holloway RG, Gramling R, Kelly AG: Estimating and communicating prognosis in advanced neurologic disease. Neurology 2013, 80:764-772.

10.1186/cc13144

Cite this article as: Gilmore and Sheth: Improving ascertainment and communication of prognosis in intracerebral hemorrhage. Critical Care 2013, 17:1021 